\title{
Performance Assessment of Coded-Beam High Throughput Satellites
}

\author{
Eman S. Abass' ${ }^{1}$ Joseph V. M. Halim², Hadia M. El-Hennawy ${ }^{2}$ \\ ${ }^{1}$ Electronics and Communication Department, MTI University, Cairo, Egypt \\ ${ }^{2}$ Electronics and Communication Department, Ain Shams University, Cairo, Egypt \\ Email:eng.emansalah@gmail.com,j.victor@nilesat.com.eg, helhennawy@ieee.org
}

How to cite this paper: Abass, E.S., Halim, J.V.M. and El-Hennawy, H.M. (2017) Performance Assessment of Coded-Beam High Throughput Satellites. Int. J. Communications, Network and System Sciences, 10, 218226.

https://doi.org/10.4236/ijcns.2017.108B023

Received: June 8, 2017

Accepted: August 11, 2017

Published: August 14, 2017

\begin{abstract}
High-throughput satellite (HTS) systems usually make use of Multibeam coverage at high frequency bands in order to offer broadband access of large areas. Multibeam coverage increases the available capacity through frequency reuse and spatial separation. However, one of its major drawbacks is interspot interference which considered the motivation to propose a new approach using a coding technique to distinguish between beams that will be employed to mitigate this interference without the need of frequency reuse. This approach makes the use of orthogonal codes to identify beams and allow using the total satellite bandwidth per beam. Proposed system double the bandwidth used in each beam and save the usage of antenna polarization resource used in former designs, which may be used as a multiple accessing resource inside the coded beams. The objective of this paper is to provide a comprehensive performance assessment methodology for the proposed approach. Result validation is introduced by comparing the proposed system performance with conventional systems that relay on the DVB standards.
\end{abstract}

\section{Keywords}

High-Throughput Satellites, Multibeam Satellites, Coded Beams, Walsh Coding, CDMA Satellites

\section{Introduction}

The ever increasing demand for broadband communication opens up new fecund opportunities for the next generation satellite system [1], The development of multi-spot beam networks with high number of beams for both fixed and mobile applications that support user frequencies from L to Ka-bands is a trending technology [2]. This growing demand pushes more and more for spectrum re-usage over the coverage. A coverage composed of a large number of 
spots implies the possibility of strong interference generated by all the beams reusing the same frequencies and impacting the overall performances [3], In this context, due to the non-perfect isolation among the beams, the amount of interference coming from the side-lobes of the co-channel spots may significantly impact the link budget and thus ultimately the achievable throughput. Cochannel interference becomes therefore one of the main impairments limiting the performance of the system if not properly tackled [4], moreover, the interference mitigation techniques suffer from certain degradation [5] [6] [7]. This is due to the fact that the processing must be separated in isolated processing units. In [8] the next generation of HTS allowing to reach the Terabit/s satellite systems is characterized, The targeted capacity achieved by increasing the frequency reuse factor with reduced beam sizes and increased available user link spectrum, The author proposed solutions to address the identified difficulties faced his approach. the first Challenge was the existed bandwidth scarcity, as the system total capacity is directly proportional to the accessible spectrum and radio frequency resources remain limited and subject to coordination with other terrestrial systems . Author suggests allocating the full Ka-band spectrum to the users and moving the feeder links to optical links using the $1.55 \mu \mathrm{m}$ wavelength technology. Second challenge appears with reducing beams size a large gateway network is required as well as large spectrum per beam, the Terabit/s system requires a significant uplink bandwidth up to $377 \mathrm{GHz}$. With The frequency plan that has been optimized to limit as far as possible the number of gateways by allocating large spectrum per gateway. Still, up to 37 gateways are required to serve all the users beams. This is quite a significant number of ground stations compared to more classical satellite system. As a result, the ground segment becomes an important expense category when deploying the full system. the current design of [8] assumes a regular fix design thus to provided capacity needs to users through the satellite lifetime seemed to be challenging for several reasons: User distribution over the coverage area is not and will not sustain homogeneous during satellite lifetime, also the consumption habits of users will significantly evolve during this time. As a result, some of the beams might get quickly fully loaded while others remain emptier loaded. This leads to un-optimized usage of the satellite resources. Paper assumed that the implementation of photonics technologies will allow for a significant mass reduction and to be available by 2020 time frame.

Specifically, the difficulties arise in the current approaches due to the dependency of the classical model of beam design with colors of different frequencies and polarization and regarding the frequency and the time resources only. Here an algorithms is developed to solve the underlying problems based on using the orthogonally of Code Division Multiple Access (CDMA) Walsh coding and design the multibeam system regarding codes as a new resource in the HTS systems. this solution was not proposed in the literature yet, though it showed a promising performance enhancement in the mobile communication systems, upon the usage of this resource the multibeam satellite communication systems 
will take a new shape by differentiating the beams using assigned codes while it can occupy the total satellite bandwidth, eventually here, the way to reach the Terabit/s satellite systems becomes more achievable. This scenario will be investigated throughout this paper. Figure 1 shows Illustration of a cluster with coded beams that supports the down streams from Satellite to the User Terminals (UTs) for a fixed service, each color will express a different code assigned to data transmitted within each beam, while transmission is continuous using the whole satellite bandwidth. The remaining of this paper is organized as follows. The system model is presented in Section II. Section III demonstrates the Numerical results and analysis. Finally some concluding remarks are presented in Section IV.

\section{System Model}

In the following, the satellite downlink is illustrated while satisfy the users' QoS requirements, and, in the next section, we will analyze the relevant performance. A multibeam downlink featuring $\mathrm{NM}$ beams belonging to the set $\mathrm{M}$ is considered. The term "color" is used to designate a Walsh coded beam, each user terminal (UT) is served by one beam and is tuned on the frequency carrier and its data is encoded with the beam identifying Walsh. Now Define the power received by the UT as $P_{u, i}$ for the $i$ th user in the beam $\left(i=1,2, \cdots, N_{u}-1\right)$, generally the received $E_{b} / N_{o}$ represented in [9] [10] is as follows.

$$
\left(\frac{E_{b}}{N_{o}}\right)_{u, i}=\frac{B}{R_{u}} \frac{P_{u, i}}{\sum_{K=1, K \neq i}^{N_{u}-1} P_{u, K}+I+n_{0} W}
$$

where $B$ is the spreading bandwidth; $N_{u}$ represent the number of users in a beam and $I$ is the other beam interference; and $n_{0}$ is the level of the background noise power spectral density. The following facts will be considered throughout the paper analysis:

1) While the assigning codes are orthogonal, there is no other beam interference $I=0$ in (1).

2) The path loss attenuation between the UT and the Satellite is proportional to $10^{\xi / 10} R^{-4}$, where $R$ is the distance from the UT to the Satellite and $\xi$ is a Gaussian random variable with zero mean and standard deviation $\sigma$.

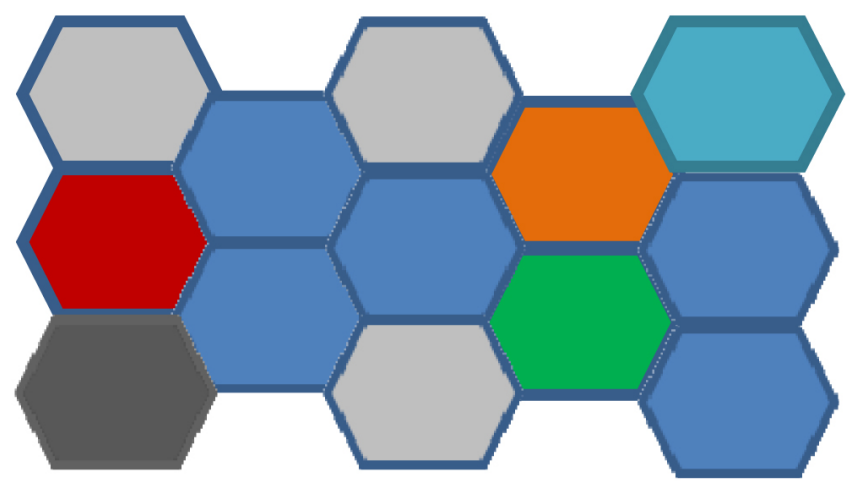

Figure 1. Illustration of a cluster with coded beams. 
3) Perfect power control mechanism is assumed due to the nature of satellite link according to this fact, $P_{u, i}=P_{u}$ for all $i$. the effect of power-control delays and errors $\sigma_{-} \mathrm{x}=0 \mathrm{~dB}$ in perfect power control, and values less than 1 reflect lower performance. Hence (1) is modified to

$$
\left(\frac{E_{b}}{N_{o}}\right)_{u, i} \cong \frac{B}{R_{u}} \frac{P_{u}}{\left(N_{u}-1\right) P_{u}+n_{0} W}
$$

For proper reception, the received $\frac{E_{b}}{N_{o}}$ should be greater than the required $\frac{E_{b}}{N_{o}}$

$$
\left(\frac{E_{b}}{N_{o}}\right)_{u} \geq\left(\frac{E_{b}}{N_{o}}\right)_{u_{r e q}}
$$

To satisfy the information data rate requirement for all beams, the following relation should be satisfied:

$$
R_{u} \geq R_{u_{\text {req }}}
$$

According to (3) and (4), the received $E_{b} / N_{o}$ that represented in (2) is limited as follows;

$$
\left(\frac{E_{b}}{N_{o}}\right)_{u_{\text {req }}} \leq\left(\frac{E_{b}}{N_{o}}\right)_{u} \leq \frac{B}{R_{u_{r e q}}} \frac{P_{u}}{\left(N_{u}-1\right) P_{u}+n_{0} W}
$$

This equation upper-bounds the number of users, $\left(N_{u_{1}}, N_{u_{2}}, \ldots, N_{u_{N-1}}\right)$ as follows;

$$
\left(N_{u}-1\right) P_{u}+n_{0} B \leq \frac{B}{R_{u_{\text {req }}}}\left(\frac{E_{b}}{N_{o}}\right)^{-1} P_{u_{\text {req }}}=(S N R)_{u_{\text {req }}}^{-1} P_{u}
$$

Applying this relation between the received signal powers within the beam, as in (6), the relation between the user numbers and the required SNR can be derive [11] as.

$$
\sum_{i=1}^{N_{u}-1} \frac{N_{u}}{(S N R)_{u_{\text {req }}}^{-1}+1} \leq 1
$$

To satisfy the requirements of all users, the numbers of users in the system, as derived in [11], are limited by;

$$
\sum_{i=1}^{N_{u}-1} \gamma_{u, i} N_{u} \leq 1
$$

Here the resource amount used by user $i$ in the beam corresponds to 1. i.e. the resources used by users should not exceed total system resource, where,

$$
\gamma_{u, i}=\frac{1}{(S N R)_{u_{i_{\text {req }}}}^{-1}+1}
$$

This equation specifies capacity plan in the $M$ dimensional space. All points $\left(N_{u, i_{1}}, N_{u, i_{2}}, \ldots, N_{u, i_{M-1}}\right)$ the hyperplane represent possible numbers of supporta- 
ble users in each beam of the multibeam system. Now considering the system reliability requirement $\beta \%$ which is defined as the predetermined value of probability that the received SNR is larger than the required SNR, $P_{r}\left(S N R \geq S N R_{r e q}\right)=\beta \%$. The outage probability $p$ is lower bounded by the required system reliability $\beta \%$ [12] as follow;

$$
p=P_{r}\left\{\sum_{i=1}^{N-1} \gamma_{u_{i}} N_{u_{i}} \leq 1\right\} \geq \beta
$$

Furthermore, it is assumed that the allocated frequency bandwidth $W$, the standard deviation of the received SNR, and the system reliability $\beta \%$ are the same for all beams. To satisfy the requirements of all users, the numbers of users in the system that were confined by (8), Equation (9) will be generally as derived in [11] as follow;

$$
\gamma_{u_{i}}=\frac{1}{\frac{B}{R_{u_{i} \text { req }}}\left(\frac{E_{b}}{N_{o}}\right)_{u_{i} \text { req }}^{-1} \frac{1}{1+f} 10^{\frac{Q^{-1}(\beta)}{10} \sigma_{x}-0.012 \sigma_{x}^{2}}+1}
$$

where $Q^{-1}$ is the inverse Q-function where Q-function is defined as $Q(x)=\int_{-\infty}^{x}\left(1 / \sqrt{2 \pi} e^{-y^{2} / 2}\right) d y$. In particular, $f$ is other cell interference factor defined as the ratio of the inter-spot interference from inter beam to the intra-spot interference from intra beam, $\left\langle\frac{1}{1+f}>\right.$ is the average value of frequency reuse factor and the quantity of $10^{\frac{Q^{-1}(\beta)}{10} \sigma_{x}-0.012 \sigma_{x}^{2}}$ indicates the effect of the imperfect power control error and the system reliability $\beta \%$ on the system capacity. Applying this on the proposed scheme will give,

$$
\gamma_{u_{i}}=\frac{1}{\frac{B}{R_{u_{\text {ireq }}}}\left(\frac{E_{b}}{N_{o}}\right)_{u_{\text {i req }}}^{-1} 10^{\frac{Q^{-1}(\beta)}{10}-0.012}+1}
$$

So several variables that determine system capacity can be summarized as follow:

- $B$ is the spreading bandwidth;

- $R_{u_{\text {ireq }}}$ is the required information data rate;

- $\beta \%$ is system reliability;

- $\left(\frac{E_{b}}{N_{o}}\right)_{\text {req }}$ is the required bit energy-to-noise power spectral density ratio.

In practice, the capacity bounds may change with variations of the system capacity parameters, so it is important to consider the effect of the disturbance of system parameters. Imperfections encountered in CDMA systems in most cases are due to imperfect power control error which is negligible here due to the satellite environment, and the effect of which on CDMA capacity is practically linked to system reliability. In this paper we focused on quantitatively describing the change of system capacity due to the previous parameters with consideration of system reliability that is considered as an example of sensitivity analysis in CDMA systems. 


\section{Numerical Results and Analysis}

This work focuses on the usage of CDMA coding over multibeam satellite network, the performance of the proposed scheme is assessed using (6), (8) and (12) with the system introduced in [13], first to indicate the information bit rate supported by the system for every user in each beam with the circumstances applied in [13]. Typically, a bandwidth of $1.05 \mathrm{GHz}$ in Ka band is considered in the BATS system of [2] with 302 beams over Europe, and the DVB-RCS2 MODCODs [14] are applied. And using the equation describing CDMA throughput [15] is used.

$$
R_{u_{\text {ireq }}}=\frac{B \times m}{(1+\rho) G_{p}}
$$

where $B$ is the total satellite bandwidth, $\mathrm{m}$ is the modulation order and is set to $2,3,4,5,6$ referring to [14], $\rho$ is the roll-off factor for the filtering and typically considered by $0.35,0.25,0.2$ [14], and $G_{p}$ is the processing gain and is dependent on number of beams as the proposed system showed, for an instance there will be 512 code required to identify the 302 beam as [13] so $G_{p}$ will equal 9, nevertheless. Table 1 and Table 2 illustrate the information bit rate with various modulation techniques, roll-off factors and processing gains, using parameters of [13] and suggested number of beams as [2].

The values above is achieved without using interference cancellation (IC) algorithms and without scheduling and availability of $99.99 \%$ thus consider a promising contribution over the previous conventional systems that relay on DVB standards and apply IC and scheduling algorithms to improve the capacity.

Table 1. The information bit rate with various modulation techniques, roll-off factors and processing gains, $\mathrm{B}=1.05 \mathrm{GHz}$.

\begin{tabular}{|c|c|c|c|c|c|c|c|}
\hline \multicolumn{8}{|c|}{ Using 302 beam as assumed in [13] } \\
\hline \multicolumn{3}{|c|}{$\rho=0.35$} & \multicolumn{3}{|c|}{$\rho=0.25$} & \multicolumn{2}{|r|}{$\rho=0.2$} \\
\hline \multirow{5}{*}{$R_{u_{\text {ireq }}}$} & 172.839 Mbps & QPSK & & 186.666 Mbps & QPSK & & 194.444 Mbps QPSK \\
\hline & 259.259 Mbps & 8-PSK & & $279.999 \mathrm{Mbps}$ & 8-PSK & & 291.666 Mbps 8-PSK \\
\hline & $345.788 \mathrm{Mbps}$ & 16-PSK & $R_{u_{\text {ireq }}}$ & 373.332 Mbps & 16-PSK & $R_{u_{\text {iveq }}}$ & 388.888 Mbps 16-PSK \\
\hline & $432.098 \mathrm{Mbps}$ & 32-PSK & & 466.666 Mbps & 32-PSK & & $486.111 \mathrm{Mbps} 32$-PSK \\
\hline & $518.518 \mathrm{Mbps}$ & 64-PSK & & $560 \mathrm{Mbps}$ & 64-PSK & & 583.333 Mbps 64-PSK \\
\hline
\end{tabular}

Table 2. Total system capacity for all the beams, with $\mathrm{B}=1.05 \mathrm{GHz}, \beta=99.99 \%$ for carrier power $=-74 \mathrm{dBm}$ parameters of [13].

\begin{tabular}{|c|c|c|c|c|c|c|c|c|}
\hline \multicolumn{9}{|c|}{ Using 302 beam as assumed in [13] } \\
\hline \multicolumn{3}{|c|}{$\rho=0.35$} & \multicolumn{3}{|c|}{$\rho=0.25$} & \multicolumn{3}{|c|}{$\rho=0.2$} \\
\hline \multirow{5}{*}{$C$} & $0.498 \mathrm{Tbps}$ & QPSK & & 0.538 Tbps & QPSK & & 0.560 Tbps & QPSK \\
\hline & 0.746 Tbps & 8-PSK & & 0.806 Tbps & 8-PSK & & 0.840 Tbps & 8-PSK \\
\hline & 0.996 Tbps & 16-PSK & $C$ & $1.075 \mathrm{Tbps}$ & 16-PSK & $C$ & $1.120 \mathrm{Tbps}$ & 16-PSK \\
\hline & 1.245 Tbps & 32-PSK & & 1.344 Tbps & 32-PSK & & 1.400 Tbps & 32-PSK \\
\hline & $1.556 \mathrm{Tbps}$ & 64-PSK & & $1.680 \mathrm{Tbps}$ & 64-PSK & & $1.750 \mathrm{Tbps}$ & 64-PSK \\
\hline
\end{tabular}


Table 3. The information bit rate with various modulation techniques, roll-off factors and processing gains, $\mathrm{B}=1 \mathrm{GHz}$.

\begin{tabular}{|c|c|c|c|c|c|c|c|c|}
\hline \multicolumn{9}{|c|}{ Using 256 beam } \\
\hline \multicolumn{3}{|c|}{$\rho=0.35$} & \multicolumn{3}{|c|}{$\rho=0.25$} & \multicolumn{3}{|c|}{$\rho=0.2$} \\
\hline \multirow{5}{*}{$R_{u_{\text {ireq }}}$} & 194.444 Mbps & QPSK & & 209.999 Mbps & QPSK & & 218.749 Mbps & QPSK \\
\hline & 291.666 Mbps & 8-PSK & & 314.999 Mbps & 8-PSK & & $328.124 \mathrm{Mbps}$ & 8-PSK \\
\hline & 388.888 Mbps & 16-PSK & $R_{u_{\text {ireq }}}$ & 419.999 Mbps & 16-PSK & $R_{u_{\text {ireq }}}$ & 437.499 Mbps & 16-PSK \\
\hline & 486.111 Mbps & 32-PSK & & $524.999 \mathrm{Mbps}$ & 32-PSK & & $546.874 \mathrm{Mbps}$ & 32-PSK \\
\hline & $583.333 \mathrm{Mbps}$ & 64-PSK & & $630 \mathrm{Mbps}$ & 64-PSK & & $656.250 \mathrm{Mbps}$ & 64-PSK \\
\hline
\end{tabular}

Table 4. Total system capacity for all the beams, with $\mathrm{B}=1.05 \mathrm{GHz}, \beta=99.99 \%$ for carrier power $=-74 \mathrm{dBm}[13]$.

\begin{tabular}{|c|c|c|c|c|c|c|c|c|}
\hline & \multicolumn{8}{|c|}{ Using 256 beam } \\
\hline & \multicolumn{2}{|l|}{$\rho=0.35$} & \multicolumn{3}{|c|}{$\rho=0.25$} & \multicolumn{3}{|c|}{$\rho=0.2$} \\
\hline & 0.422 Tbps & QPSK & & $0.456 \mathrm{Tbps}$ & QPSK & & 0.475 Tbps & QPSK \\
\hline & 0.633 Tbps & 8-PSK & & $0.684 \mathrm{Tbps}$ & 8-PSK & & 0.712 Tbps & 8-PSK \\
\hline \multirow[t]{3}{*}{ C } & 0.844 Tbps & 16-PSK & C & 0.912 Tbps & 16-PSK & C & 0.95 Tbps & 16-PSK \\
\hline & 1.055 Tbps & 32-PSK & & $1.139 \mathrm{Tbps}$ & 32-PSK & & $1.187 \mathrm{Tbps}$ & 32-PSK \\
\hline & $1.318 \mathrm{Tbps}$ & 64-PSK & & $1.423 \mathrm{Tbps}$ & 64-PSK & & $1.483 \mathrm{Tbps}$ & 64-PSK \\
\hline
\end{tabular}

Table 3 and Table 4 illustrate the information bit rate and system capacity with various modulation techniques, roll-off factors and processing gains using the parameters of [16] and reducing beam number strategy from the model suggested in [2] to decrease the processing gain and allow a wider band width per user which results an increase of the information rate and total system capacity.

Thus the average system capacity of $1.076 \mathrm{Tbps}$ is greater than the maximum value reached in [13] 766 Gbps by about 50\% above with a less complex, cost effective and interference immune system that transmit over the total satellite bandwidth saving the polarization resource.

In [17], various interference effects (CCI, ACI and XPI) and thermal noise have been discussed, and numerical results for SNIR, bandwidth efficiency and throughput have been presented over 200 beams with $1 \mathrm{GHz}$ Bandwidth and parameters included in the DVB-S2X, applying these parameters to the proposed scheme using $G_{p}=8, \rho=0.05$, typical value of $\mathrm{SNR}=18 \mathrm{~dB}$ results user information rate per beam equals to $249.999 \mathrm{Mbps}$ and system throughput of 1.00655 Tbps which is higher than the maximum value achieved in [17] (the 420 Gbps for the use of three frequencies and use of dual polarizations in each beam (3FDP) with interference cancellation of about $70 \%$ of interference ) by $140 \%$ above, each user Occupies triple the bandwidth used in the 3FDP scheme without wasting polarization resource.

Another paper [16] presented several capacity enhancing techniques, SGD techniques increases the system availability with a significant increase in the system cost by adding extra gatways, while full frequency reuse with CCI cancellation and scheduling provides the highest system capacity of $600 \mathrm{Gbps}$ in the sake 
of the availabity that decreased to $98.82 \%$.

\section{Conclusion}

In this work, a new approach presented in multiple spot beam satellite systems employing a coding technique to identify beams without the need of frequency reuse and methods of mitigating the interference effects. A new method of improving the performance, capacity and users bit rates has been proposed, and the expected benefits have been discussed and compared with other promising techniques. The preliminary results show that incorporating Spreading spot beams with CDMA Walsh coding has the potential to improve system capacity and individual user information. It is recognized, however, that this improved performance did not come at the expense of neither increased complexity nor extra bandwidth utilization as the previous work did. This system configurations and operational parameters under which the combination of coded beams with the various multiple accessing techniques, can be investigated to provide maximum scope for improved overall satellite communications system performance.

\section{Acknowledgements}

Thanks to my supervisors for their precious guiding, inspiration and support.

\section{References}

[1] Bem, D.J., Wieckowski, T.W. and Zielinski, R.J. (2000) Broadband Satellite Systems. IEEE Communications Surveys \& Tutorials, 3, 2-15. https://doi.org/10.1109/COMST.2000.5340721

[2] (2012) Broadband Access via Integrated Terrestrial and Satellite Systems (BATS), ICT-2011.1.1 BATS D4.1: Satellite Network Mission Requirements, European Project. Tech. Rep.

[3] Suffritti, R., Privitera, N., Dimitrov, S., Katona, Z., Boccolini, G., Jaeckel, S., Raschkowski, L., Kyrgiazos, A., Evans, B., Rodriguez, J.M., Yun, A., Vidal, O. and Inigo, P. (2014) On Interference Management Techniques in Broadband Satellite Systems. In: Proc. of Ka Conference.

[4] Erich, L. (2015) Towards the Terabit/s Satellite-Interference Issues in the User Link.

[5] Joroughi, V., Vazquez, M.A. and Perez-Neira, A. (2014) Multiple Gateway Precoding with Per Feed Power Constraints for Multibeam Satellite Systems. Proceedings of 20 th European Wireless Conference, May 2014, 1-7.

[6] Zheng, G., Chatzinotas, S. and Ottersten, B. (2012) Multi-Gateway Cooperation in Multibeam Satellite Systems. Proceedings of 2012 IEEE 23rd International Symposium, Sept 2012, 1360-1364. https://doi.org/10.1109/PIMRC.2012.6362558

[7] Devillers, B., Perez-Neira, A. and Mosquera, C. (2011) Joint Linear Precoding and Beamforming for the Forward Link of Multi-Beam Broadband Satellite Systems. Proceedings of Global Telecommunications Conference, 2011 IEEE, Dec 2011, 1-6. https://doi.org/10.1109/GLOCOM.2011.6133895

[8] Inigo, P., et al. (2014) Review of Terabit/s Satellite, the Next Generation HTS Systems. Proc. of 7 th Advanced Satellite Multimedia Systems Conference 2014, Livorno.

[9] Sampath, A., Kumar, P.S. and Holtzman, J.M. (1995) Power Control and Resource 
Management for a Multimedia CDMA Wireless System. IEEE Proc. of International Symposium on Personal, Indoor, and Mobile Radio Communications, 1995, 21-25. https://doi.org/10.1109/PIMRC.1995.476272

[10] Viterbi, A.M. and Viterbi, A.J. (1992) Erlang Capacity of a Power-Controlled CDMA System. IEEE Journal on Selected Areas in Communications, 892-900.

[11] Koo, I., et al. (1997) A Generalized Capacity Formula for the Multimedia Traffic. Proc. of Asia-Pacific Conference on Communications, 1997, 46-50.

[12] Gilhousen, K.S., et al. (1991) On the Capacity of a Cellular CDMA System. IEEE Trans. on Vehicular Technology, 303-312. https://doi.org/10.1109/25.289411

[13] Svilen, D., et al. (2015) Capacity Enhancing Techniques for High Throughput Satellite Communications. Springer International Publishing.

[14] (2012) Second Generation DVB Interactive Satellite System (DVB-RCS2); Part 2: Lower Layers for Satellite Standard, Digital Video Broadcasting (DVB) Std. ETSI EN, 301545-2.

[15] Roddy, D. (2006) Satellite Communications. 4th Edition, McGraw-Hill Professional.

[16] Katona, Z., Clazzer, F., Shortt, K., Watts, S., Lexow, H.P. and Winduratna, R. (2016) Performance Cost Analysis and Ground Segment Design of Ultra High Throughput Multi-Spot Beam Satellite Networks Applying Different Capacity Enhancing Techniques. Int. J. Satellite Commun. Netw., 34, 547-573.

[17] Lutz, E. (2015) Towards the Terabit/s Satellite-Interference Issues in the User Link. International Journal of Satellite Communications and Network, 34, 461-482. https://doi.org/10.1002/sat.1114

\section{Scientific Research Publishing}

Submit or recommend next manuscript to SCIRP and we will provide best service for you:

Accepting pre-submission inquiries through Email, Facebook, LinkedIn, Twitter, etc. A wide selection of journals (inclusive of 9 subjects, more than 200 journals)

Providing 24-hour high-quality service

User-friendly online submission system

Fair and swift peer-review system

Efficient typesetting and proofreading procedure

Display of the result of downloads and visits, as well as the number of cited articles

Maximum dissemination of your research work

Submit your manuscript at: http://papersubmission.scirp.org/

Or contact ijcns@scirp.org 